\title{
Situación bucal de pobladores con discapacidades en el distrito de San Luis - Lima
}

Soto-Veliz R, Vallejos-Ragas R, Campos-Sánchez R, Ouslanova P, Agüero-Cortez Z, BernalGaray N, Camargo-Hurtado J, De la Cruz-Calvo C. Situación bucal de pobladores con discapacidades en el distrito de San Luis - Lima. Rev Estomatol Herediana. 2009; 19(1):18-20.

\section{RESUMEN}

Objetivo: Establecer el estado de la salud bucal de personas con diversas discapacidades, residentes en un distrito de clase media-baja de la ciudad de Lima. Materiales y métodos: Se examinó a residentes registrados en la Oficina Municipal de Atención de la Persona con Discapacidad OMAPED, de San Luis, como parte de la primera fase del desarrollo del Sistema de atención Odontológico Especializado a implementar. La clasificación utilizada, incluía la discapacidad a) Motora b) Sensorial y c) Mental. La información bucal recabada, fue el CPOD, apiñamiento dentario (Van der Linden), el Î́ndice de Higiene Oral simplificado de Greene y Vermillion (IHOs), y la presencia de bolsas periodontales, piezas extruidas y atrición dental. Resultados: El total examinado fue de 102 personas, considerando válidos finalmente 89. Las edades oscilaron entre 6 y 93 años. Del total, el 42,2\% presentaban discapacidad motora; 35,3\% discapacidad mental y el 9,8\% discapacidad sensorial. El CPOD, reflejaba un incremento progresivo con el paso de los años, siendo mas pronunciado a partir de los 51 años, donde se observó, el valor mínino de 18 y el máximo de 32. Entre los componentes del CPOD, las piezas perdidas prevalecieron en los 3 grupos de discapacidad, siendo mayor en los subgrupos motor y sensorial. También llama la atención que las obturaciones eran muy escasas. Conclusiones: La condición bucal de las personas con discapacidad se deteriora rápidamente con la edad.

Palabras clave: ATENCIÓN DENTAL PARA PERSONAS CON DISCAPACIDADES / CARIES DENTAL / SALUD BUCAL / HIGIENE BUCAL.

Dental Status of people with special needs from the District of San Luis - Lima ABSTRACT

Objective: To establish the dental status of people with special needs, who live in a district of a Low-Middle Economical class in Lima. Materials and methods: People registered at the office for people with special needs of the City Hall of District of San Luis (OMAPED) were examined. This study is a first stage of the development of an Specialized System of Dental Care. The classification of the special needs included: a) mobility handicap, b) Sensorial handicap; c) mental handicap. The dental status included information of DMF-t; crowding (Van der Linden classification), Oral Hygiene Green Vermillion index, presence of periodontal pockets, extruded teeth, and dental attrition. Results: A total of 102 people with special needs were examined, only 89 were considered in the data. Age ranged from 6 to 93 years old. $42.2 \%$ of the total examined people had mobility handicap, $35.5 \%$ had mental handicap, and $9.8 \%$ had sensorial handicap. DMF-t showed a progressive rise as people grew older, it reached its highest value at the age of 51, with a minimum value of 18 and a maximum value of 32 . Regarding the components of DMF-t, missing teeth component was more frequent in the three groups of handicapped; the highest values were observed in the motor and sensorial handicap. It also calls the attention the very few restored teeth. Conclusions: The dental status of people with special needs deteriorates rapidly with aging.

Key words: DENTAL CARE FOR DISABLED / DENTAL CARIES / ORAL HEALTH / ORAL HYGIENE.

\section{Introducción}

Según estudios realizados en el Perú, se calcula que el $45,4 \%$ de la población está afectada por algún tipo de deficiencia, el 31,28\% tiene alguna discapacidad y 13,08\% alguna minusvalía (1).

La discapacidad en orden de prevalencia señalan que la invalidez se presenta en el $28,05 \%$, ceguera $20,86 \%$, sordera $14,37 \%$, retardo mental 12,39\%, alteraciones mentales $9,79 \%$ y mudez 6,68\%.

En el Perú, la necesidad de atención referente a problemas de salud bucal de la población con discapacidad se ha reflejado en algunas investigaciones, entre ellas la de Falconí et al. (2), que estudió a niños con parálisis cerebral, encontrando que el $80 \%$ de ellos presentan abrasión dental, 90\% hipoplasia del esmalte y el 98\% tiene un riesgo alto de caries dental. Así mismo, Soto et al. (3) en un estudio de personas que acudían a tres centros especializados en atención a personas con discapacidad de la ciudad de Lima y Cusco, concluyó que la prevalencia de caries fue de 92,35\%, mientras que el CPOD fue de 7,4 y el ceod de 8,4. Es importante

\section{Rosario Soto Veliz ${ }^{1}$ Raúl Vallejos Ragas ${ }^{1}$ Rosario Campos Sánchez ${ }^{2}$ Polina Ouslanova ${ }^{1}$ Zoraida Agüero Cortez ${ }^{3}$ Noel Bernal Garayar ${ }^{3}$ Jesús Camargo Hurtado ${ }^{3}$ Carolina De la Cruz Calvo ${ }^{3}$}

'Instituto de Desarrollo Odontológico - IDEO Docente de Universidad Federico Villarrea Bachiller en Odontología

\section{Correspondencia}

Rosario Soto Veliz

Jr. Trinidad 203 Urb. Villa Jardín - Lima 30, Perú e-mail: charoteeth@gmail.com

Recibido : 8 de enero del 2009

Aceptado : 05 de mayo del 2009 señalar que el CPOD, en personas de 12 años sin discapacidad en el Perú está alrededor de 3,7 (4).

En los últimos años, la condición de la salud bucal en personas con discapacidades, se ha visto afectada por factores tales como a) La desinstitucionalización de estas personas (modelo de atención dentro de su propia comunidad, iniciado en 1960), b) Incremento de la supervivencia, c) Escaso entrenamiento profesional odontológico y d) Inadecuado financiamiento de servicios dentales (5). 
A pesar del elevado porcentaje de personas con discapacidad, la información relativa a su salud bucal, es aún escasa. El propósito del presente estudio fue establecer el estado de la salud bucal de personas con diversas discapacidades, residentes en un distrito de clase media-baja de la ciudad de Lima.

\section{Material y métodos}

El estudio, correspondió a la primera fase del desarrollo del Sistema de Atención Odontológico Especializado a implementar en el Distrito de San Luis de la Provincia de Lima, por parte del Instituto de Desarrollo Odontológico - IDEO, en coordinación con el Municipio de San Luis. Esta fase comprendía el diagnóstico basal de la condición de salud bucal de las personas con discapacidad, residentes en el mencionado distrito.

La selección de las personas a examinar, se basó en el registro que contaba la Oficina Municipal de Atención de la Persona con Discapacidad - OMAPED, de San Luis. La evaluación se realizó en forma domiciliaria, entre abril y agosto del 2008, fecha en la que se completó la evaluación de la totalidad de los registrados. Toda evaluación fue realizada previo consentimiento de la persona con discapacidad o familiar a cargo, según sea el caso.

Para el examen se elaboró una ficha de registro, que incluía el tipo de discapacidad, el mismo que era informado por la misma persona o el familiar responsable. La clasificación utilizada, incluía la discapacidad a) Motora ${ }^{a}$ b) Sensorial $^{\mathrm{b}}$ y c) Mentalc (6). No se consideró al grupo de enfermedades crónicas degenerativas de esta clasificación.

Entre la información bucal recabada, se registró el índice de caries dental CPOD, apiñamiento dentario (Van der Linden), el Índice de Higiene Oral simplificado de Greene y Vermillion (IHOs), y la presencia de bolsas periodontales, piezas extruídas y atrición dental.

Para el índice de CPOD, se utilizó el criterio propuesto por la OMS (7). Definiendo un diente cariado ante la presencia de una cavidad evidente, un socavado en el esmalte o reblandecimiento del tejido dentario. Se utilizó para el diagnóstico clínico iluminación artificial para la observación visual con el uso de un explorador dental.

Previo al inicio del estudio, se realizaron reuniones de coordinación y entrenamiento entre los observadores, con el fin de homogenizar las observaciones, así como, considerar la forma de abordaje en los domicilios donde se realizó el estudio. En los casos de no encontrar a las personas en su domicilio, se repitió la visita hasta por tres veces, antes de descartar al paciente.

\section{Resultados}

Se evaluaron un total de 102 personas, considerando válidos finalmente 89. Las edades oscilaron entre 6 y 93 años. Del total, el 42,2\% presentaban discapacidad motora; $35,3 \%$ discapacidad mental y el 9,8\% discapacidad sensorial.

El déficit motor se presentaba mayormente en los grupos de edad, entre 6 y 10 años y en los mayores de 61 años. El grupo de personas con déficit mental se incrementaba porcentualmente entre los 11 y 60 años. Se hallaron más mujeres
(54,45\%) que varones (45,55\%).

$\mathrm{Al}$ considerar al total de personas, se observó que el CPOD, reflejaba un incremento progresivo con el paso de los años, siendo mas pronunciado a partir de los 51 años, donde se observó, el valor mínimo de 18 y el máximo de 32. El CPOD, del subgrupo con discapacidad motora fue de 22, el sensorial 19 y el mental 15.

Entre los componentes del CPOD, las piezas perdidas prevalecieron en los tres grupos de discapacidad, siendo mayor en los subgrupos Motor y Sensorial. También llama la atención que las obturaciones eran muy escasas.

El índice de placa (IHOs), reveló que el valor promedio fue de 1,87 , equivalente a un calificativo de deficiente. En el subgrupo con discapacidad motora, se observó un valor más elevado $(1,94)$, equivalente también a la calificación de deficiente. Los otros dos subgrupos presentaron valores de 1,8 respectivamente.

$\mathrm{La}$ presencia de bolsas periodontales osciló entre el 5,6\% y $10 \%$ en los tres grupos de discapacidad. Las piezas extruidas fueron mas frecuentes en el grupo de discapacidad motora. Finalmente la atrición dental, fue mayor en el grupo de discapacidad sensorial.

\section{Discusión}

Vivir con una discapacidad es un desafío, mas aú $\mathrm{n}$ en un país como el Perú, donde el $39.3 \%$ son calificados como pobres (gasto diario de una persona equivale a US\$2,50), estando el 19,4\% de los limeños en esta condición, distribuidos en diversos distritos entre ellos San Luis (8). Sobre la población con 
discapacidad en Lima Metropolitana, se considera que sólo el 33\% tiene actividad laboral, de éstos el 50\% tiene una remuneración menor de US\$150.00 al mes y el $62 \%$ no cuenta con seguro de salud (9).

Entre las discapacidades incluidas en el presente estudio, resalta que el segundo grupo con mayor prevalencia observado sea el de discapacidad mental, muy por encima de la discapacidad sensorial, esto a diferencia de la prevalencia nacional señalada en el censo nacional del 2003 (1), donde el grupo al que consideramos con discapacidad sensorial (ciegos, sordos y sordomudos), ocupaba el segundo lugar. Los criterios de selección y clasificación de discapacidades generalmente son amplios y complejos, pudiendo este factor el que influyera en estas diferencias.

Con relación a los rangos de edad, se observó que la discapacidad motora fue incrementándose progresivamente con la edad, coincidente con la Encuesta de Hogares sobre Discapacidad de Lima Metropolitana y Callao del 2005 (EHODIS) (9), que señala que la discapacidad se incrementa con la edad. Sobre el sexo, el estudio encontró mayor número de mujeres que hombres, mientras que la Encuesta de Hogares, no encontró diferencia.

El índice de caries dental (CPOD), reflejó un preocupante incremento con la edad, siendo la pérdida de las piezas dentarias el componente más elevado. Esto se podría deber a que la extracción dental es la alternativa de tratamiento mas común y al alcance de una población con discapacidad, con insuficientes recursos económicos y en su mayoría sin seguro de salud (9). Es importante resaltar el escaso número de establecimientos de salud con servicios de rehabilitación (75 en todo el país), estimando según nuestros resultados preliminares, que menos de la mitad cuentan con servicio odontológico (10).

La presencia de placa bacteriana, es el factor determinante en el desarrollo de caries y enfermedad periodontal, alcanzando valores preocupantes en todos los subgrupos. Estos resultados son coherentes con los resultados de Falconí (2) y Soto (2), quienes observaron alto riesgo de caries dental y la prevalencia de caries del 92.35\% respectivamente.

Los resultados muestran que bajo una perspectiva odontológica, los diversos tipos de discapacidad presentan problemas diferenciados $y$ por lo tanto, requieren intervenciones particulares que reviertan la crítica situación bucal en la que se encuentran.

Por lo tanto, se hace necesario desarrollar programas preventivos odontológicos, dirigidos a evitar la perdida de piezas dentarias, que afectarían progresivamente la calidad de vida de estas personas.

\section{Agradecimientos}

A la licenciada Elizabeth Caballero, responsable de la OMAPED del distrito de San Luis, por su constante apoyo en el estudio.

\section{Referencias bibliográficas}

1. INEI. Censo Nacional de Población y Vivienda - Perú de 1993.

2. Falconí E, Huanqui M, Lay S, Paredes F, Tanco R. Problemática Odontológica del niño con Parálisis Cerebral. Odont. Pediátrica Noviembre 2004 - Abril 2005 3(1): 16-20
3. Soto R, Vallejos R, Falconí E, Monzón F. Patologías bucales en niños con Encefalopatía Infantil en el Perú. Rev Estomatol Herediana. 2006; 15(1):115-9.

4. MINSA. Documento técnico Plan Nacional de Salud Bucal "Sonríe Siempre Perú". RM No 516-2008 / MINSA. 21 Julio del 2008.

5. Fenton S, Hood H, Holder M, May P, Mouradian W. The American Academy of Developmental Medicine and Dentistry: Eliminating Health Disparities for Individuals with Mental Retardation and other development Disabilities. J Dent Educ. 2003; 67(12): 1337-44

6. Tan Castañeda Nuvia, Rodríguez Calzadilla Amado. Correspondencia entre la formación académica del estomatólogo relacionado con pacientes especiales y la práctica estomatológica integral. Rev Cubana Estomatol [periódico en la Internet]. 2001 Dic [citado 2008 Nov 01] ; 38(3): 181-191. Disponible en: http://scielo.sld.cu/ scielo.php?script=sci_arttext\& pid=S0034-75072001000300006 \&lng=es\&nrm=iso.

7. WHO. Oral health surveys: basic methods. 4th ed. Geneva: World Health Organization. 1997.

8. INEI. Informe Técnico: La pobreza en el Perú en el año 2007.

9. INEI - CONADIS. Encuesta Nacional de Hogares sobre Discapacidad en Lima Metropolitana y Callao EHODIS 2005.

10. Arroyo J. Derecho a la salud de las personas con discapacidad. 1era edición. Lima: Fondo editorial del congreso del Perú. 2006. 